\title{
REPLY TO JOHN LU
}

\author{
John P. Doll, Emil H. Jebe, and Robert D. Munson \\ University of Missouri, University of Michigan, \\ and American Potash Institute, Inc.
}

\begin{abstract}
7 HE procedures presented in our paper are applicable only to least I squares estimation equations linear in the unknown parameters. While we attempted to mention wherever appropriate that the equations we were using were linear, we never specifically mentioned linearity as a prerequisite. Mr. Lu has clarified this-however, we doubt that careful readers would be misled by the article as it stands.

The use of transformations in statistical procedures leads to a number of considerations that we did not wish to deal with in our article. Readers who prefer the Cobb-Douglas equation, or other exponential equations involving logarithmic transformations, would be interested in the work of $\mathrm{J}$. Neyman and E. L. Scott, "Correction for Bias Introduced by a Transformation of Variables," Annals of Mathematical Statistics, 31:643-55, 1960, as well as the article by Carter and Hartley.
\end{abstract}

